perform an initial univariate logistic regression, followed by a multivariate analysis to identify predictor variables associated with adverse events such as recurrent ED visits, and admission to hospital or ICU for hyperglycemia within 30 days. We will include individual patients who have multiple recurrent visits to the ED during the study period and statistically weight for these using generalized estimating equations (GEE), which are used to develop regression models for correlated data that arise from repeated measures of the same individuals over time. Finally, a clinical risk tool will be derived by rounding the beta coefficients. Internal validation will be conducted using bootstrapping techniques. Importance: ED visits for hyperglycemia significantly affect both the healthcare system overall and the individual patient. The results of this project will assist clinicians to better identify these patients and enable them to intervene either medically or educationally to prevent subsequent visits to the ED. As a result, patients will have improved care, better blood glucose control, and be identified for closer follow-up with a family physician or diabetes specialist. Furthermore, by aiming to reduce the number of recurrent visits, this project may reduce ED utilization and the associated healthcare costs with frequent visits and admissions for hyperglycemia.

\section{Moderated Poster Presentations}

\author{
MP01 \\ The canary in the coal mine: Does palliative care consultation \\ influence emergency department utilization? \\ Z. Polsky, BSc, E. Lang, MD, A. Sinnarajah, MD, T. Fung, PhD, \\ B. Thomas, $\mathrm{PhD}$, University of Calgary, Calgary, $\mathrm{AB}$
}

Introduction: For cancer patients undergoing active treatment, emergency department (ED) visits may be an indicator of a breakdown in continuity and quality of care. Palliative care (PC) may be an important resource for patients in need of symptom management even during treatment with curative intent. This study aims to describe ED utilization by cancer patients and determine if PC consults impact ED use. Methods: Patient data from the Tom Baker Cancer Center (TBCC) was linked to PC and ED data as a retrospective cohort study. ED data was obtained from two administrative databases and PC data was obtained from four administrative databases and restricted to the first four hundred days following diagnosis. Univariate and Multivariate analyses were used. Results: Three actively treated cancer patient cohorts were identified based on first presentation following intake at the TBCC: 1) Used ED first ( $n=1637), 2)$ Used PC first ( $n=539)$, and 3 ) Only used services at the TBCC $(n=2153)$. Using Multivariate analysis, patients living alone or who had a diagnosis of prostate or breast cancer were more likely to access the ED first or to only use services at the TBCC rather than access PC first. Patients who were divorced, on income support, or diagnosed with a lung or GI cancer, were more likely to access PC first rather than access the ED or only use services at the TBCC. A subgroup analysis was performed on those who accessed the ED at some point during their care, consisting of three groups: 1) ED Only Users $(n=1091)$, 2) ED First Users, who also accessed PC $(n=546)$, and 3) PC First Users, who also accessed the ED. There was a significant difference in rates of ED visits between the three groups: ED Only Users went to the ED at a rate of 3.8 per 1000 patient days; ED First Users, who also accessed PC, went to the ED at a rate of 7.7 per 1000 patient days; and PC First Users, who also accessed the ED, went to the $\mathrm{ED}$ at a rate of 9.2 per 1000 patient days $(\mathrm{p}<0.001)$. Conclusion: In a tertiary cancer centre, patients who were divorced, on income support, or diagnosed with lung or GI cancer were more likely to access PC. Amongst those patients who presented to the ED, those who accessed PC first had higher rates of ED use. Further explorations of presenting complaints, utilization patterns, and symptom burdens will be analyzed to determine if early PC consults can influence or decrease ED utilization.

Keywords: palliative care, cancer patients, utilization

\section{MP02}

Paramedic safety culture across Eastern Ontario

J.E. Sinclair, MScN, P. Price, MMgt, M.A. Austin, MD, A. Reed, BSc, BPE, MSc, MD, E.S. Kwok, MD, Regional Paramedic Program for Eastern Ontario, Ottawa, ON

Introduction: Safety culture is defined as the shared beliefs that an organization's employees hold relative to workplace safety. Perceptions of workplace safety culture within paramedic services have been shown to be associated with patient and provider safety outcomes as well as safe work practices. We sought to characterize paramedics' perceptions of the organizational safety culture across Eastern Ontario, Canada to provide important benchmarking data to evaluate future quality initiatives. Methods: This was a cross-sectional survey study conducted September 2015-January 2016 in 7 paramedic services across Eastern Ontario. We distributed an abridged version of Patterson's previously published EMS-SAQ survey, measuring six domains of workplace safety culture, to 1,066 paramedics during continuing medical education sessions. The questions were presented for rating on a 5 point Likert scale $(1=$ strongly agree, $5=$ strongly disagree $)$ and a response of 1 or 2 was considered a 'positive perception' response. We present descriptive statistics and chi-square tests where appropriate. Results: We received responses from 1,041 paramedics (97.6\%), with a response rate varying between $88.0 \%$ and $100 \%$ across the 8 paramedic services. One third (33.6\%) were Advanced Care Paramedics (ACPs) and 39.4\% of paramedics had more than 10 years' experience. The percentage of positive responses for each domain were: Safety Climate $31.2 \%$ (95\% CI 28.4-34.1), Teamwork Climate $29.3 \%$ (95\% CI 26.6-32.1), Stress Recognition 56.8\% (95\% CI 53.8-59.8), Perceptions of Management $67.0 \%$ (95\% CI 64.0-69.8), Working Conditions $42.6 \%$ (95\% CI 39.6-45.7), Job Satisfaction $41.6 \%$ (95\% CI 38.6-44.6). Primary care paramedics had more positive perception responses for Job Satisfaction ( $45 \%$ vs $35 \%, p=0.002$ ), whereas ACPs had more positive perception responses for Stress Recognition $(61.5 \%$ vs $54.1 \%$, $\mathrm{p}=0.022$ ). No association was found between gender or years of experience and a positive perception of any safety domain. Conclusion: The results provide valuable workplace safety culture data that will be used to target and evaluate needed quality improvement initiatives while also raising some awareness to paramedics of important factors related to patient and provider safety.

Keywords: paramedic, safety culture, patient safety

\section{MP03}

Predicting survival after pediatric out-of-hospital cardiac arrest I. Drennan, BSc, K. Thorpe, MMath, S. Cheskes, MD, M. Mamdani, MPH, MA, PharmD, D. Scales, MD, PhD, A. Guerguerian, MD, L.J. Morrison, MD, MSc, Rescu, St. Michael's Hospital, Toronto, ON

Introduction: Pediatric out-of-hospital cardiac arrest (OHCA) is unique in terms of epidemiology, treatment, and outcomes. There is a paucity of literature examining predictors of survival to help guide resuscitation in this population. Objective: The primary objective was to examine predictors of survival to hospital discharge. The secondary objective was to determine the probability of return of spontaneous circulation 
(ROSC) over the duration of resuscitation. Methods: We performed a retrospective cohort study of non-traumatic OHCA ( $<18$ years) treated by EMS from the Toronto Regional RescuNET Epistry-Cardiac Arrest database from 2006 to 2015 . We used competing risk analysis to calculate the probability of ROSC over the duration of resuscitation. We then used multivariable logistic regression to examine the role of Utstein factors and duration of resuscitation in predicting survival to hospital discharge. Candidate variables were limited to Utstein factors and duration of resuscitation due to the number of events. We used area under the receiver operating characteristic (ROC) curve (AUC) to determine the predictive ability of our logistic regression model. Results: A total of 658 patients met inclusion criteria. Survival to discharge was $10.2 \%$ with $70.1 \%$ of those children having a good neurologic outcome. The overall median time to ROSC was $23.9 \mathrm{~min}$. (IQR 15.0,36.7). However, the median time to ROSC for survivors was significantly shorter than the time to ROSC for patients who died in hospital (15.9 (IQR 10.6 to 22.8 ) vs. 33.2 (IQR 22.0 to 48.6); P value $<0.001)$. There was a decrease in the odds of survival of $14 \%$ per minute during the first 25 minutes of cardiac arrest. Older age (OR 0.9, $95 \%$ CI $0.86,0.99$ ), and longer duration of resuscitation (OR 0.9, 95\% CI $0.88,0.93)$ were associated with worse outcome while initial shockable rhythm (OR 5.8, 95\% CI 2.0,16.5), and witnessed arrests (OR $2.4,95 \%$ CI $1.10,5.30$ ) were associated with improved patient outcome. The AUC for the Utstein factors was fair (0.77). Including duration of resuscitation improved the discrimination of the model to 0.85 . Conclusion: Inclusion of duration of resuscitation improved the performance of our model compared to Utstein factors alone. However, our results suggest there are a number of other important factors for predicting patient outcome from pediatric OHCA.

Keywords: pediatric, cardiac arrest, resuscitation

\section{MP04}

Interim analysis of the impact of the Emergency Department Transformation System on ambulance offload delay

S. Pawa, MBChB, K. Van Aarsen, MSc, A. Dukelow, CHE, MD,

D. Lizotte, PhD, M. Zheng, London Health Sciences Centre, London, ON

Introduction: Emergency Department Systems Transformation (EDST) is a bundle of Toyota Production System based interventions implemented in two London, Canada tertiary care Emergency Departments (ED) between April 2014 and July 2016 to improve patient care by increasing value and reducing waste. Some of the 17 primary interventions included computerized physician order entry optimization, staff schedule realignment, physician scorecards, and a novel initial assessment process. Offload delays are associated with longer hospital length of stay and delayed admission, and may increase morbidity and mortality. Delays also result in fewer circulating ambulances in the community. CIHI sets a benchmark of 30 minutes as an acceptable offload target. It is possible that EDST may have impacted offload times. Methods: Middlesex-London EMS provided offload times. Data was collected from London Health Sciences Centre including daily ED visit volumes, ED occupancy, offload nursing hours, and site variation. A binomial logistic regression analysis was performed to determine the impact of interventions and confounding variables on the proportion of patients meeting CIHI benchmark. A chi-square analysis was done comparing proportion of patients meeting the benchmark in the first 3 months versus the last 3 months to identify overall impact of EDST to date. Results: Increased offload nursing hours had a positive impact $(p<0.001)$ on the proportion of offload times meeting the CIHI benchmark while increased ED visit volume and hospital inpatient volume had a significant negative impact $(\mathrm{p}<0.001)$. At both ED sites, the proportion of patients meeting the offload target ranged from $58-83 \%$ over the timeframe. There was a significant increase in the proportion of patients meeting the benchmark from the first quarter to the last quarter (69.6\% vs $75.0 \%$; $95 \%$ CI $3.45 \%$ to $7.38 \%, p=0.000$ ). Specific interventions had varying degrees of impact on offload times. Conclusion: The proportion of patients meeting the benchmark offload time varied over the study timeframe but significantly increased with EDST implementation. Offload times are one of many outcomes we aim to improve with EDST and it remains an ongoing process as new interventions continue to be implemented. Once transformation is complete, future studies will focus on the impact of EDST on all ED flow metrics, and patient and provider satisfaction.

Keywords: emergency department systems transformation (EDST), ambulance, offload

\section{MP05}

Do emergency department staff use a current domestic violence documentation tool or other forms of intimate partner violence documentation in patient records?

J. Vonkeman, BSc, P.R. Atkinson, MD, J. Fraser, BN, R. McCloskey, MN, PhD, Dalhousie Medicine New Brunswick, Saint John, NB

Introduction: Domestic violence (DV) rates in smaller cities been reported to be some of the highest in Canada. It is highly likely that emergency department staff will come across victims of intimate partner violence (IPV) in their daily practice. The purpose of this study is to better understand current practices for detecting IPV as we are currently uncertain whether patients are assessed for IPV and what the current documentation practices are. Methods: A standardized retrospective chart review, following principles outlined by Gilbert et al. 1996, was completed by two researchers to capture domestic violence documentation rates in patients presenting to the ED between January and April 2015 with injuries that may have been caused by IPV. To assess self-reported documentation/questioning practices, a cross-sectional online survey was distributed to ED staff via staff email lists three times between July and October 2016, with a response rate of $45.9 \%$ $(n=55)$. The primary outcome was DV field usage. Secondary outcomes included documentation in patient charts and current questioning habits. Results: Overall, we found documentation in $4.64 \%$ of all included patient charts $(\mathrm{n}=366)$. No documentation was noted in the DV field. $52.4 \%$ patients with deliberate injuries had no documentation of assailant identity. With regards to self reported documentation practices, $16.4 \%$ of ED staff never questioned female patients about intimate partner violence, $83.6 \%$ asked when thought appropriate, and none asked routinely. None of the staff used a structured screening tool. $60 \%$ of ED staff documented their questioning but $92.7 \%$ did not use the DV-field for documentation. $58.2 \%$ of ED staff could not identify the DV field and $45.5 \%$ of respondents did not know how to interpret the DV field if positive. Conclusion: Our findings suggest that the current documentation tool (DV-field) is not being utilized. Furthermore, low rates of IPV documentation, and potentially questioning, in high risk patients indicates that there is need to improve current practises.

Keywords: intimate partner violence, screening, emergency department

\section{MP06}

Use of ultrasound and $x$-ray to predict improvement in hip osteoarthritis symptoms following intra-articular steroid injection

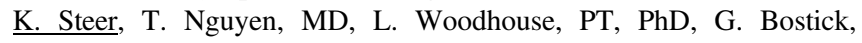
PT, PhD, B. Smith, MD, J. McGoey, R.G. Lambert, MB, J. Jaremko, $\mathrm{MD}, \mathrm{PhD}$, University of Alberta, Edmonton, $\mathrm{AB}$ 